\title{
19. ROTATION OF THE EARTH (ROTATION DE LA TERRE)
}

President: H. M. Smith.

Vice-President: C. Sugawa.

Organizing Committee: E. P. Fedorov, B. Guinot, Wm. Markowitz, P.J. Melchior, R. W. Tanner, R. O. Vicente, S. Yumi.

\section{INTRODUCTION}

The past three years have been marked by steady improvements in the utilisation of classical techniques employing conventional optical instruments such as PZT, astrolabe and transit instrument. There has also been a rapidly growing interest in the investigation of relationships between the astronomically-observed motions of the Earth and the data available from geophysical studies. In addition, and perhaps more significant to the future programme of work in the field of Commission 19, has been the continuing development of new techniques such as lunar laser ranging; very-long base-line interferometry (VLBI); laser, Doppler, and photographic tracking of artificial satellites.

Indications of the wide range of interests in the rotation of the Earth were the high attendance and large number of scientific papers at IAU Symposium No. 48 held at Morioka, Japan in 1971. This is an opportunity to place on record the thanks and appreciation of all participants to our Japanese colleagues for the excellent arrangements made. The published proceedings form a valuable survey of the present state of the subject.

Analyses of the conventional astronomical observations for the determination of time and of the variation of latitude have been carried out at the Bureau International de l'Heure and have permitted the introduction of an improved weighting system and the determination of correction terms for Earth tides and for the effects of diurnal nutation.

In a recent paper J. Kovalevsky (Mitt. Astron. Ges. 31, 49-60, 1972) has proposed the establishment of a chain of observatories, well distributed over the surface of the Earth, and equipped with the full range of instruments for optical and radio observations of UT1 and polar motion and for the recording of Earth tides and other relevant geophysical phenomena.

For the efficient planning of such projects, and for the fuller utilisation of data from all existing series of observations, there is an urgent need to strengthen international cooperation, particularly in view of the growing demand for precise results with the minimum delay.

The rapid service introduced by the BIH for the dissemination of up-to-date data on UT1 and the polar coordinates is dependent on a temporary financial arrangement by a national agency. It will be necessary to consider the extent of the continuing need for such a service, and the means by which it might be supported.

\section{CHANGES IN INSTRUMENTATION}

Lists of the instruments collaborating in international programmes are published in the Annual Reports of the BIH and of the IPMS.

Calgary: a new PZT is due to replace the existing instrument at the beginning of 1973 .

Paris: the astrolabe prism has been replaced by an optical square, insensitive to temperature variations; a new differential drive has also been fitted (1).

Cagliari: a new VZT is scheduled to commence operating on the parallel $39^{\circ} 08^{\prime} 12^{\prime \prime}$ in the spring of 1973.

Mizusawa: a new PZT was installed in 1972 in an underground air-conditioned room $(\mathrm{a}=25 \mathrm{~cm}$, $\mathrm{f}=354 \mathrm{~cm})(2)$. 
Dresden: a new circum-zenithal instrument has been brought into service (3).

Japan: an automatic electronic astrolabe has been constructed at the Geographical Survey Institute (4).

\section{RESEARCH IN INSTRUMENTATION}

Research into methods of improving conventional instruments continues. A new $65 \mathrm{~cm}$ PZT is now being developed for use by the U.S. Naval Observatory. In Belgrade great improvements in the accuracy of the VZT have been achieved by the reduction of thermal effects (5) and the methods used in Blagovestchensk to obtain such high quality latitude observations by the use of a zenith telescope merit careful study. The effects of instrumental errors and atmospheric refraction in the results of the two new photo-electric transit instruments are being studied at Pulkova, where experiments are in progress with a photo-electric micrometer. Detailed studies of PZT plate motions have been made in Japan (6) and the Herstmonceux PZT is being fitted with a moire fringe displacement transducer for improved monitoring of the plate motion. Improvements in astrolabes have been effected in Japan (7) and at Paris, where a photo-electric micrometer is being developed.

\section{REVISION OF OBSERVING CATALOGUES AND OF EARLIER OBSERVATIONS}

New catalogues with improved star co-ordinates were introduced in Washington (1970), Richmond (1972) and Ottawa (1972). Proper motion errors in the adopted values used in the CalgaryHerstmonceux catalogue have been deduced from the Herstmonceux PZT observations made in the years 1958-71 and, taking these errors into account, new values of the adopted mean places of the catalogue stars are being computed from a joint analysis of the observations at the two stations. At Tokyo intercomparisons of different PZT systems of star co-ordinates have been made by the use of the SAO catalogue (8) and the Tokyo Meridian Department is currently engaged in a programme of observation of PZT stars for 14 northern hemisphere observatories (9). A new catalogue of right ascensions which has been found to give a more precise system than FK4 or $\mathrm{N} 30$ has been derived for 807 stars observed in 9 time service stations of the U.S.S.R. (10). New reductions of observations and discussions of the results have been carried out for the Greenwich FZT (Cookson telescope) for the years 1911-36 by R. d'E. Atkinson, and for the Pulkova latitude observations (1948-67) by V. Sakharov (11). A comprehensive review of the Washington PZT results has been carried out by D. McCarthy.

Improved proper motions and declinations for ILS catalogue stars have been reduced by P. Melchior and $\mathrm{R}$. Dejaiffe and their results have given rise to much research on the polar motion and on catalogue comparisons of their results (12).

\section{RESEARCH IN PROBLEMS CONCERNED WITH THE ROTATION OF THE EARTH}

Limitation in space makes it impossible to catalogue in full all the research, based both on the classical methods of astronomy and the new techniques, that has been published in the past three years. Current problems are comprehensively treated in papers published in

(1) Earthquake Displacement Fields and the Rotation of the Earth, 1970, Ed. L. Mansinha, D. E. Smylie, and A. E. Beck, Reidel Publishing Co., Holland - the proceedings of a NATO Advanced Study Institute, University of Western Ontario, June 1969.

(2) Rotation of the Earth, 1972, Ed. P. Melchior and S. Yumi, Reidel Publishing Co., Holland the proceedings of IAU Symposium No. 48 held at Morioka, May 1971.

(3) Extra Collection of Papers contributed to IAU Symposium No. 48 (1972), Ed. S. Yumi (Sasaki Printing and Publishing Co., Japan).

Reports have been received of research published in many scientific journals but adequate references have not always been supplied.

At Potsdam effects of wind on seasonal variation have been studied in a discussion on the changes in the rotation of the Earth in the years 1956-69 (13), and at Dresden temperature effects 
in the observations have been examined (14). A. Mikhailov has carried out research on the polar motion and on how the results are affected by an error in the constant of precession (15). At Basle, J. O. Fleckenstein is comparing the PZT observations of Neuchatel with the transit observations made at Milan in an investigation of the colour effect in the constant of aberration. From a study of early ILS results G. Teleki has demonstrated the importance of "refractional star pairs" and he urges their inclusion in future ILS programmes (16). The observatories of Belgrade and Warsaw share a common meridian and advantage is being taken of this situation for closer co-operation in latitude observations (17).

The observatories of Paris, Brussels and San Fernando are now engaged in artificial satellite observations. G. Billaud reports that by minimising effects of errors in the lunar ephemeris, studies of the Chandler Wobble by different methods have produced accordant results, indicating occasional abrupt changes in the amplitude of the Wobble (18). Effects of atmospheric circulation in the rotation of the Earth (19) have also been studied at Paris where investigations of diurnal nutation and zonal tides have also been carried out (20). N. and A. Stoyko have continued their research in diverse fields of interest relating to the polar motion and the rotation of the Earth (21). The long period terms in polar co-ordinates and continental drift have been studied by E. Proverbio (22), and E. Fichera has made a contribution to the Working Group on polar co-ordinates.

C. Sugawa has submitted a long report on current research in Japan, the most important aspects of which appear to be concerned with the following topics: Extensive investigation of the Chandler Wobble, the results of which indicate that there are two fundamental periods with physical significance in the motion. These periods, 1.18 and 1.22 years, are associated with the mantle and core respectively. Sundry other theoretical investigations of the polar motion have been carried out and, in discussions of observations, tentative explanations have been offered for the discrepancies between the polar motions derived independently from time and latitude observations (23). Studies have also been made of the differences between ILS and Doppler measures of the polar motion (24). R. O. Vicente and S. Yumi have computed polar coordinates, referred to the CIO from 1889-1968 (25). The $Z$ terms in the ILS data have been analysed by C. Sugawa and 7 terms, with periods ranging from 8 to 54 months, have been identified in the observations. Observational results have also been examined for the effects of atmospheric pressure, motions and refraction. S. Iijima and S. Okazaki have shown that the annual term is the only periodic term common to both the polar motion and the rotation of the Earth (26).

At Hamburg, H. Enslin has determined the coefficients in the semi-diurnal tidal wave $\left(M_{2}\right)(27)$; these are about half the amplitude of those discovered by N. O'Hora in the results of the Herstmonceux PZT (28). The enhanced effects at Herstmonceux are now being investigated as also are the fortnightly terms in the results. L. V. Morrison has completed an analysis of 30000 lunar occultations of stars for the period 1955.5 to 1972.0 and has established a revised ET2 scale which corresponds closely with the IAT scale over the period (29).

\section{ADDENDUM}

Shortly after the above report was sent to press a comprehensive account of progress and developments in the U.S.S.R. was published. This account was not received in time for inclusion in the main report but special arrangements were made for the publication of the following precis, with references.

Investigations in the statistics of observations include studies in the resolution of discrete lines in the power spectrum of latitude observations (30), the smoothing of observations using Whittaker's methods (31) with separate studies on the selection of optimum control parameters (32) for use in this work. The more important analyses of the observations discuss

(a) the reality of the supposed diurnal nutation,

(b) whether the Chandler wobble consists of two components.

In investigations of the diurnal nutation A. I. Emetz and Ya. S. Yatskiv discovered, by analysis of the Washington (1916-1940) and Pulkovo (1905-1941) latitude observations, that instead of 
the single term with period 204 days that is predicted by theory, the results are affected by four significant terms with periods 219, 211, 204-205 and 194 days. Analysis of the polar motion from 1846-1900 by Ya. S. Yatskiv, A. A. Korsun and L. V. Rykhlova has failed to reveal two periods in the Chandler wobble. Contradictory results have been obtained in investigations of the regularity of the period of the wobble. A. A. Mikhailov supports the notion that the period is not constant; he has concluded (33) that the length of the period and the amplitude of the disturbance are positively correlated but results by other investigators (34) indicate that the period is constant.

Attempts have been made to discover the geophysical basis of the polar motion and different results have been obtained. One suggestion (35) attributes the secular polar motion to a shift of the whole of the Earth's crust (or at least the whole of the northern hemisphere) but evidence (36) has also been derived from time observations that indicates relative motions of continental blocks.

The directions of the axes of co-ordinates of the empirical or conditional reference system, defined by the time and latitude observations of a number of independent observatories, depends on the conditions imposed in combining the observations and research (37) has been carried out on the most suitable conditions that should be employed. By the use of tidal charts a value of $3.8 \mathrm{~ms} /$ century has been deduced (38) for the retardation of the Earth's rotation.

\section{REFERENCES}

1. 05.032 .002 .

2. Iwadate, K. 1972, Proc. Int. Lat. Obs. Mizusawa, 12, 54-61 (in Japanese).

3. Steinert, K. G. Geod. Geophys. Veröff. III, 20.

4. Tsubokawa, I., Hokugo, S. 1971, Proc. IAU Symp. 48.

5. Milovanovic, V. S. IAU Symp. 48 (Extra papers).

6. Yoshinari, M., Fujiwara, K. 1972, Tokyo Astr. Obs. Rep. 16, 1 (in Japanese).

7. Yokoyama, K., Sakai, S., Iwadate, K. Proc. Int. Lat. Obs. Mizusawa, 11, 109-114 (in Japanese).

8. Iijima, S., Niimi, Y., 1971, Ann. Tokyo Astr. Obs. XII, 4.

9. Yasuda, H., Kamijo, I. 1971, Ann. Tokyo Astr. Obs. XIII 1.

10. Pavlov, N. N., Afanasjeva, P. M., Staritsyn, G. V. 1971, Trudy. Pulk. Obs. LXXVIII 4-59.

11. Sakharov, V. (in print).

12. (a) Melchior, P. J., Dejaiffe, R. Ann. Obs. R. Belg. 3e serie, X 3, 277.

(b) Dejaiffe, R., Melchior, P. J. $1971, A$. \& A. 14, 468-472.

Melchior, P. J., Dejaiffe, R., Verbeiren, R. 1971, Proc. IAU Symp. 48.

13. 05.044 .033 and 04.044 .012 .

14. Dittrich, J., Wächter, S. (in press).

15. Mikhailov, A. A. 1971, Russ. A. J. 1 and 6.

16. 05.045 .011 .

17. 03.045 .012 .

18. 04.044 .003 .

19. Cazenav, A., Lambeck, K. $\quad$ 1972, Trans. Am. Geophys. Un. 53, 342.

20. 01.045.001, 04.081.049.

21. $04.044 .023 ; 04.045 .041 ; 04.044 .035$.

22. (a) Proverbio, E. et al. 1971, IAU Symp. 48.

(b) Proverbio, E. Quesada 1972, Ann Geofisica, XXV, 37.

23. (a) 05.045 .015 .

(b) Takagi, S. 1972, Pub. Int. Lat. Obs. Mizusawa, VIII, 1, 1-29.

(c) Yasuda, H., Aoki, S. $\quad$ 1972, Pub. astr. Soc. Japan, 24, 127.

24. Sugawa, C., Kakuta, C. $\quad$ 1972, Proc. Int. Lat. Obs. Mizusawa, 12, 39-45.

25. Vicente, R. O., Yumi, S.

(a) 1969, Pub. Int. Lat. Obs. Mizusawa, VII, 1, 41-50.

(b) 1970, ibid. VII, 2, 109-112.

26. Iijima, S., Okazaki, S. 1972, Pub. astr. Soc. Japan, 24, 109.

27. Enslin, H. 1971, Vermessungstechnik, 19, 2.

28. O'Hora, N. P. J. Phys. Earth Planet Int. (in press).

29. Morrison, L. V. $\quad$ Nature (in press).

30. Godisov, N. P., Vasylyev, O. B. 1971, Extra collection of papers contr. to IAU Symp., 48, 123. 
31. Fedorov, E. P., Glagoleva, I. I. 1962, Dopovidy AN U.S.S.R., 4, 473.

32a. Yatskiv, Ya. S. 1969, Voprosy Astrometrii, 84, Kiev.

32b. Sakharov, V. I., Vasilyev, O. B. 1971, Extra collection of papers contr. to the IAU Symp., $48,113$.

33. Mikhailov, A. A. 1971, Astr. Zu., 48, No. 6.

34. Nesterov, V. V., Rykhlova, L. V. 1970, ibid., No. 2.

35. Mikhailov, A. A. 1970, ibid., No. 3.

36. Pavlov, N. N. 1970, ibid., No. 4.

37a. Fedorov, E. P. et al. 1972, IAU Symp., 48, 78.

37b. Fedorov, E. P. et al. 1972, Dvyzhenie polusov Zemly s 1890.0 po 1969.0, Kiev.

38. Pariisky, N. N. et al. 1972, IAU Symp., 48, 240.

REPORT OF THE INTERNATIONAL POLAR MOTION SERVICE

\section{General}

More than 50 stations and observatories all over the globe have cooperated in the work of the International Polar Motion Service during this period consecutive with the earlier years. They observed latitude, time and latitude, or time only, with various kinds of instruments such as the zenith telescope, the photographic zenith tube, the astrolabe and the transit instrument.

The Central Bureau of the IPMS collected the data every week or every month and calculated the coordinates of the pole every month from the data obtained at the 5 ILS stations. The preliminary results were summarized every month in the Monthly Notes of the IPMS as a rapid service and the detailed definitive values of the results were published every year in the Annual Report of the IPMS, the last volume of which was the one for 1970 issued in 1972.

The coordinates of the pole have been calculated so far only from the results of latitude observations made at the 5 ILS stations. Studies have been made on how best to combine all the observational data of time and latitude in order to derive the most probable pole coordinates: many problems still remain, such as errors in star positions, station coordinates, the definition of mean latitudes, and the appreciable discrepancies between pole coordinates derived independently from the time and latitude data.

\section{Results of the ILS during 1949.0 to 1962.0}

The ILS results for the period from 1949.0 to 1962.0 derived by Prof. G. Cecchini have already been compiled ready for publication in a complete form similar to earlier volumes. Prof. A. Marussi, president of the Italian Geodetic Commission, is considering photographing each page of Prof. Cecchini's manuscript; a final decision will be made soon. It is not certain, however, that the Italian Geodetic Commission will meet all the publication charges.

\section{Cooperative observation of time and latitude with the PZT}

(a) Northern parallel of latitude of $39^{\circ} 8^{\prime}$.

It was recommended by the IAU at its General Assembly in Prague 1967 that each of the 5 ILS stations on the parallel of $39^{\circ} 8^{\prime}$ north should be equipped with PZT's to observe time and latitude in parallel with the observation of latitude by the visual instruments.

Mizusawa has already been equipped with a PZT and a second PZT was completed on the same parallel in May 1972.

Kitab is going to be equipped with a PZT which will be moved from Pulkovo Observatory. Necessary modifications on the mechanical parts are being made in Pulkovo Observatory.

The Italian Geodetic Commission has decided to install a new PZT on $39^{\circ} 8^{\prime}$ north, but not in Carloforte. The proposed site is very near to Cagliari, Sardinia.

The National Ocean Survey, National Oceanic and Atmospheric Administration of the United States proposes to install two PZT's, one at Gaithersburg and the other at Ukiah, but it is understood that there may be some delay owing to difficulties of financing. 